OPEN ACCESS

Edited by:

Ivan V. Litvinov,

McGill University, Canada

Reviewed by:

Abdulhadi Hazzaa Jfri,

McGill University, Canada

Ximena Wortsman,

University of Chile, Chile

Elizabeth Ann O'Brien,

McGill University, Canada

*Correspondence:

John W. Frew

jfrew@rockefeller.edu

Specialty section:

This article was submitted to Dermatology,

a section of the journal

Frontiers in Medicine

Received: 23 July 2019 Accepted: 21 October 2019 Published: 06 November 2019

Citation:

Grand D, Navrazhina K and Frew JW (2019) A Scoping Review of Non-invasive Imaging Modalities in Dermatological Disease: Potential Novel Biomarkers in Hidradenitis Suppurativa. Front. Med. 6:253. doi: 10.3389/fmed.2019.00253

\section{A Scoping Review of Non-invasive Imaging Modalities in Dermatological Disease: Potential Novel Biomarkers in Hidradenitis Suppurativa}

\author{
David Grand ${ }^{1,2}$, Kristina Navrazhina ${ }^{1,3}$ and John W. Frew ${ }^{1 *}$ \\ ${ }^{1}$ Laboratory of Investigative Dermatology, The Rockefeller University, New York, NY, United States, ${ }^{2}$ Albert Einstein College of \\ Medicine, Bronx, NY, United States, ${ }^{3}$ Weill Cornell/Rockefeller/Sloan Kettering Tri-Institutional MD-PhD Program, Weill \\ Cornell University, New York, NY, United States
}

Background: The development of imaging-based biomarkers has the potential to overcome major challenges in the accurate and reproducible assessment of disease severity and response to novel therapies in Hidradenitis Suppurativa (HS). Understanding the advantages and limitations of existing non-invasive imaging modalities in dermatological disease will aid in the development of hypotheses and inform the design of future studies.

Methods: A scoping review was performed using Medline, Embase, Web of Science Databases and evaluation of "gray literature" until June 30, 2019. Citations were examined according to pre-defined inclusion and exclusion criteria. Citations were reviewed by two independent reviewers. Narrative Synthesis was used to summarize data, structured by imaging modality.

Results: Non-invasive imaging modalities, such as ultrasound, MRI, RCM, EIS, OCT, and MIT, were identified. Only ultrasound, MRI and MIT have been used in HS. Image modalities vary in image depth, resolution, cost, accessibility and correlation with known aspects of disease activity in HS.

Discussion and Conclusion: The benefits and limitations of each imaging modality are products of cost, accessibility, validity and reliability. An additional hurdle to the development of image-based biomarkers in HS is a lack of established analytical benchmarks that can be correlated with existing biological, inflammatory and clinical parameters. This review has identified potential imaging biomarkers, as well as relevant analytical benchmarks that reflect the presence or absence of disease. Further investigation work is needed to analytically and clinically validate these imaging variables in order to identify potential imaging biomarkers in HS.

Keywords: hidradenitis suppurativa, acne inversa, ultrasound, non-invasive, confocal microscopy, electrical impedance, MRI 


\section{INTRODUCTION}

The degree of interest in non-invasive imaging modalities in dermatology has increased dramatically over the last decade. Given that visual inspection alone is limited in evaluating disease extent, histopathological assessment of skin through the use of biopsies remains a vital part of a dermatologist's armamentarium (1). Non-invasive imaging modalities have the potential to compliment, and in some instances, replace, the need for skin biopsies. Although this prospect is promising, it is dependent upon whether the specific aspect of the image, known as the imaging biomarker, is both analytically and clinically valid (through assessment of sensitivity, specificity, accuracy, precision, and other relevant performance characteristics against a gold standard test and a pre-defined clinical endpoint) (2) and whether the process of obtaining the imaging biomarker is cost effective and easily administered (3). The field of oncology demonstrates successful use of imaging biomarkers in diagnosis and therapeutic monitoring (3). Well-established guidelines exist for the development and validation of such biomarkers. Imagingbased biomarkers are desirable for diseases that demonstrate the following qualities: a disconnect between clinical examination and the disease status of the patient; a lack of a validated, reliable blood or serum biomarker; and a cost-effective reproducible method of imaging, the results of which will provide a clinical benefit to the patient at a pre-defined endpoint.

Hidradenitis Suppurativa (HS) is a chronic inflammatory disorder which is often clinically underestimated in terms of disease extent, thereby necessitating the use of imaging modalities such as ultrasound to map disease extent. Validated and/or reliable blood/serum-based biomarkers do not currently exist. The identification, development and validation of imagingbased biomarkers in HS has the potential to improve diagnostic and disease severity assessments. If validated against predefined disease characteristics, these imaging modalities have the potential to be developed into therapeutic and predictive biomarkers of clinical response. This is particularly desirable given issues with poor reliability of clinical assessments (4) and elevated placebo response rates in clinical trials (5). Whilst many tests are routinely utilized as diagnostic biomarkers in other areas of medicine (e.g., prostate-specific antigen) and predictive/safety biomarkers have been routinely used in dermatology (e.g., TPMT polymorphisms prior to Azathioprine therapy and G6PD polymorphisms prior to Dapsone therapy) (6), it is unclear which aspects of non-invasive imaging modalities have been identified and/or analyzed for their potential as biomarkers of dermatologic disease.

\section{Aims}

We undertook a scoping review of the literature pertaining to non-invasive imaging modalities in dermatologic disease with a particular focus on aspects of imaging that may be applicable to HS. The aim of a scoping review is to "map the body of literature pertaining to a research question" (7). Scoping reviews are particularly useful in emerging fields where the aim is to "identify knowledge gaps, scope a body of literature or...clarify concepts" (8). They differ from systematic reviews in that the overall aims of the review encompass a broader field of inquiry, whilst still adhering to rigorous pre-defined methodology (8). The outcomes of a scoping review may be used to inform a further systematic review, or develop concepts amenable to further research and investigation.

\section{Methods}

This scoping review was developed using previously described methodological framework of Peters et al. (9) Literature search was undertaken to identify papers regarding non-invasive imaging modalities in dermatologic disease. A particular focus was made on inflammatory dermatoses, particularly on HS. Inclusion criteria were all study designs, any language, any imaging technique, all age groups, sex, ethnicity, healthcare settings, and treatment modalities. Exclusion criteria included animal studies, and ex vivo imaging studies.

\section{Search Strategy}

Databases searched included MEDLINE (1946-June 30, 2019), Embase (1980-June 30, 2019) and Web of Science (1990-June 30, 2019). Gray literature was searched using Google Scholar and the first 100 results to the developed search strategy. Terms included in the search strategy included (skin) AND (imaging OR ultrasound OR MRI OR impedance OR confocal) AND (in vivo) AND (diagnosis OR biomarker) AND (inflammation).

\section{Study Selection}

All identified citations from databases $(n=884)$ and the first 100 citations in the gray literature $(n=100)$ were manually examined, and duplicates removed by one author (DG). Two authors (DG and JF) independently reviewed the titles and abstracts from the remaining papers $(n=372)$. All papers referring to non-human studies were excluded $(n=169)$. The remaining articles $(n=203)$ were screened through examination of the full text, with an additional 47 articles excluded as not pertaining to dermatological disorders. The inclusion or exclusion of citations was based upon unanimous agreement by the two independent reviewers with disagreements mediated by referral to a third independent reviewer for mediation $(\mathrm{KN})$. Citations were categorized according to type of imaging modality employed.

\section{Data Extraction}

Data was extracted by two independent reviewers (DG and JF) with data collated and presented as a narrative synthesis categorized by imaging modality.

\section{RESULTS}

A total of 122 manuscripts were included in review. A PRISMA diagram outlining the literature review process is presented in Figure 1. Six main imaging modalities were identified: Ultrasound, Magnetic resonance imaging (MRI), Reflectance Confocal Microscopy (RCM), Electrical Impedance Spectroscopy (EIS), Optical Coherence Tomography (OCT), and Medical Imaging Thermography (MIT). 


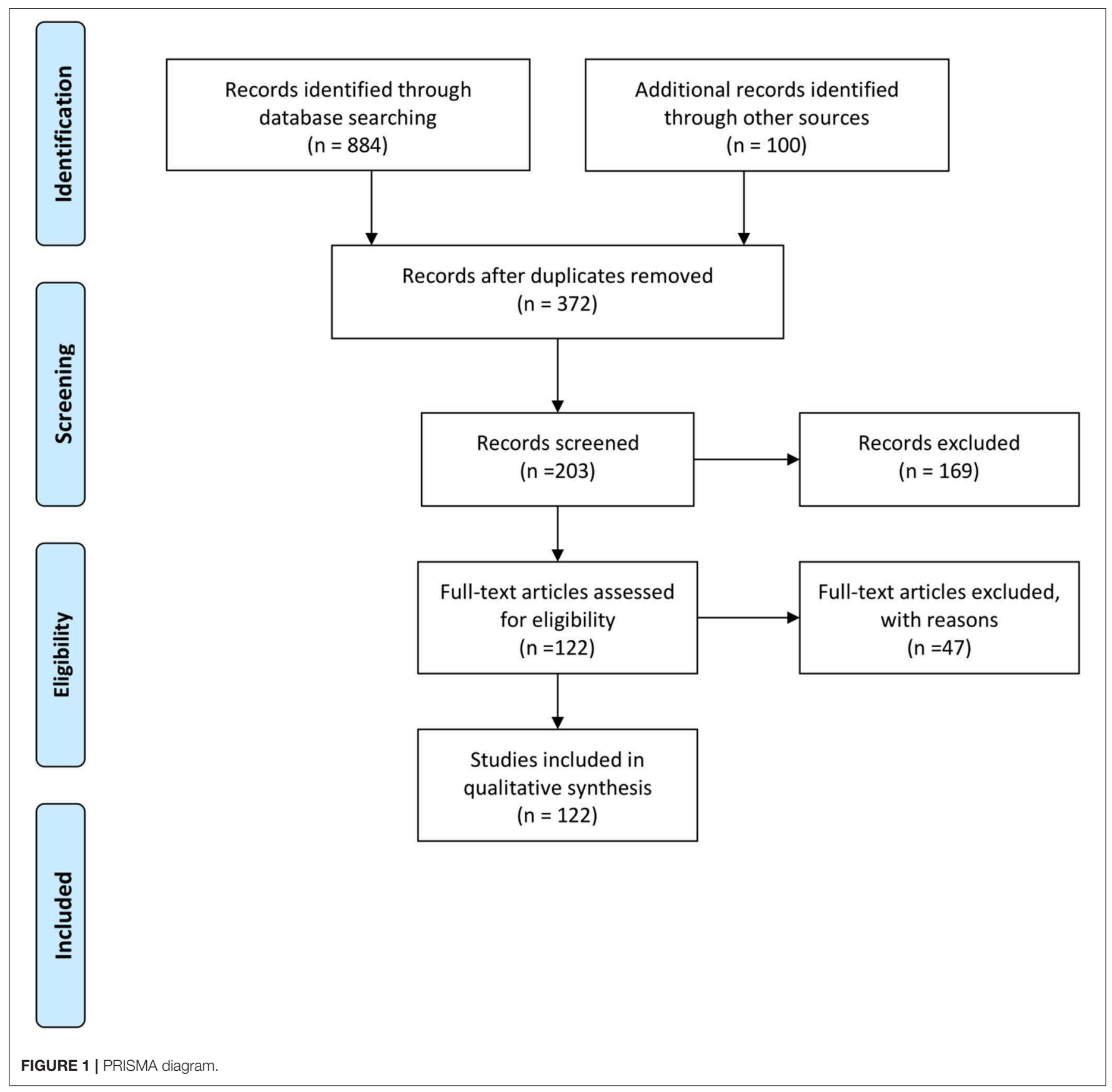

\section{Ultrasound}

Ultrasound is an affordable and portable method of imaging both epidermal characteristics such as epidermal thickness $(10,11)$ and deeper subcutaneous structures $(10,12)$. Typical ultrasound probe frequencies used for cutaneous examination range from 7.5 to $20 \mathrm{MHz}$ (11). However, higher frequency probes (50$150 \mathrm{MHz})$ can allow for more accurate representation of epidermal thickness as seen in psoriasis (12-14). The limitations of such high frequency probes are reduced penetration depth and hence lower resolution regarding deeper structures (1517), hence a combination of probe frequencies often present the best option for multiple targets in cutaneous tissues. Epidermal measurements $<0.1 \mathrm{~mm}$ are prone to inaccuracy using Ultrasound (10).

Ultrasound has been used to quantify epidermal thickness in lichen planus and psoriasis $(15,16)$. Epidermal thickness, as measured by ultrasound, demonstrates correlation with the maximum depth of rete ridges in histological specimens (17). Alterations in vessel distribution, as measured by Doppler ultrasound (18), have been correlated with metastatic potential of cutaneous malignancies (BCC, SCC, Melanoma) (19). Dermal fibrosis and adipose tissue alterations are visible and have been 
documented in HS, scleroderma and graft-vs.-host-disease (20, 21). Although color Doppler is able to provide quantitative data regarding flow rates in non-dermatological conditions [for example, echocardiography and tumor blood flow $(22,23)]$, such data is not widely validated in cutaneous inflammation with histological specimens. Additionally, Doppler ultrasound requires an informed choice of transducer and appropriate technical training in order to optimize for each patient and standardize for each visit (24). Therefore, a combination of probe frequencies allows for examining multiple targets in cutaneous tissues.

In normal tissues, the epidermis appears as a thin, hyperechoic line, whereas the dermis appears as a broad, echogenic band. Fat in the subcutaneous tissue appears hypoechoic while the surrounding fascia and connective tissue is hyperechoic (21). Sonographic studies in HS patients identified alterations in epidermal thickness (25) as broadly correlating to the well documented psoriasiform epidermal hyperplasia seen in histological sections (26). Diffuse dermal edema and inflammatory infiltrates also correspond to heterogenous hyperechoic dermal alterations in the reticular dermis. (25). Scoring systems have been proposed based upon qualitative sonographic descriptions (27) which correlate with HS disease activity, quality of life and pain scores $(28,29)$. This can be explained by the fact that visual inspection of HS lesional skin alone often underestimates the true extent of disease $(30,31)$. This fact justifies the use of sonography to define disease extent preoperatively (32).

Potential quantitative biomarkers that may be derived from ultrasound data include epidermal thickness, tunnel diameter, Doppler flow in dermal vessels and adjacent to structures including subcutaneous fluid collections. Given the well understood mechanism of Th17 immunologic axis activation and psoriasiform epidermal hyperplasia $(33,34)$, proof of concept studies into the correlation between epidermal thickness as measured by ultrasound and tissue cytokine level (such as IL-23, IL-17A, IL-17F) would aid in establishing its utility as an indirect biomarker of Th17 activity.

\section{Magnetic Resonance Imaging (MRI)}

MRI produces high resolution images of deep subcutaneous structures with specific highlighting of layers of fat-containing (T1-weighted) and water-containing (T2-weighted) content (10, 35-37). Relatively little resolution of the epidermis and dermis is seen (10), although ultrasensitive radiofrequency detectors can provide additional resolution to more superficial structures (38). Given its relatively high cost and time consumption compared to ultrasound, it is rarely utilized in dermatologic practice and research (13). The utility of MRI in dermatology is in the assessment of deep involvement of subcutaneous, fascial and muscular structures in inflammatory disorders such as morphea and sclerodermoid disorders (35), as well as in management of patients with inherited disorders such as neurofibromatosis (36) and vascular and lymphatic malformations (37).

MRI has been employed as a diagnostic modality to differentiate HS tunnels from peri-anal Crohn's disease and enterocutaneous fistulae, as well as being a pre-operative assessment tool prior to wide surgical excision of HS lesions (39). T2-enhancing cutaneous thickening, subcutaneous fat stranding, rim-enhancing abscesses, sinus tracts, and lymphadenopathy have been documented in the setting of HS (40). Structural changes (presence and dilation of dermal tunnels, number of abscesses) were documented over time suggesting a degree of sensitivity that could be used to track the progression of disease and relationship to subjective patient symptomatology (41). No documented use of radiofrequency detectors have been identified in cutaneous disease.

Given the benefits in high resolution identification of structural abnormalities in deeper tissues (10), MRI's major benefits relate to accurate identification of the disease extent, particularly in the peri-operative setting. Cost, time and the need for specialized equipment may limit the widespread adoption of MRI in the routine evaluation of HS (13). Given the documented alterations in dermal tunnel size in longitudinal imaging studies (27), dilation of dermal tunnels may have potential as an indirect marker of tunnel inflammatory activity and drainage. As current assessment outcomes (HiSCR, IHS4, AISI) (42-44) measure only the presence or absence of tunnel drainage, imaging modalities such as MRI may provide surrogate outcomes (such as tunnel diameter) which may relate to the degree of drainage. Validation of these measures could be made against pre-existing wound exudate measures and estimated volumes of discharge (based on dressing weights) in HS.

\section{Reflectance Confocal Microscopy (RCM)}

RCM provides high resolution cellular imaging and is commonly employed in the assessment and monitoring of melanocytic neoplasms (45). Relative to other imaging modalities, it is low cost. Images in the horizontal ( $\mathrm{x}$-axis) and vertical ( $\mathrm{y}$-axis) planes provide an "en face" view (45). Z-axis views can be reconstructed but the field of view is limited by a penetration depth of $\sim 200 \mu \mathrm{m}$. This roughly corresponds to the papillary dermis, limiting the utility of RCM to structural and morphological alterations of the epidermis and papillary dermis such as vascular density $(12,13)$. Additional limitations to RCM are largely dependent upon operator experience (46).

The use of RCM has been not reported in HS lesional skin; however, it has been used to identify morphological and vascular changes in other inflammatory dermatoses. Increased dermal papillary cross-sectional area, increased papillary capillary density and the presence of Munro abscesses have been shown to evolve with the clinical progression and resolution of psoriasis $(47,48)$. Comedonal structures have been characterized by RCM in acne vulgaris, as well as features preceding clinical resolution with the use of topical acne therapies $(49,50)$. Langerhan's cell and dermal dendritic cells are well visualized with RCM (51), and density variations have been noted in apocrine gland rich skin of normal individuals compared with apocrine gland poor skin (26). Psoriasiform epidermal hyperplasia (26), comedones (52) and increased dendritic cell concentrations (26) have all been identified histologically in HS lesional tissue, so examination of the morphological characteristics of HS lesional tissue using RCM could be correlated with these histological and other 
investigative findings in the future in order to ascertain if RCM has the potential to identify prospective imaging biomarkers.

\section{Electrical Impedance Spectroscopy (EIS)}

Electrical Impedance Spectroscopy (EIS) is a measurement of tissue bioimpedance. Whilst it does not produce an "image" in the traditional sense of ultrasound or MRI, it is an emerging potentially important non-invasive assessment modality. Bioimpedance reflects cellular size, shape, orientation, compaction, and cell membrane structure, and is able to reliably distinguish variations between healthy and diseased tissue (53). Currently, EIS is used as an adjuvant diagnostic tool in the setting of melanocytic lesions, producing detailed bioimpedance data which is summarized as a score from 0 to 10 . Higher scores indicate altered bioimpedance compared to within-patient control sites (11). EIS led to a change in a decision to biopsy lesions in $25 \%$ of cases, improved diagnostic accuracy, led to fewer biopsies of benign lesions and more biopsies of malignant lesions (54). It is currently unclear if EIS demonstrates altered signals in lesional HS tissue. However, given the known association of altered bioimpedance with tissue water content (55), systemic inflammation (56), and tissue catabolism in diabetes (57), it can be hypothesized that bioimpedance may be altered in HS lesional tissue due to a combination of local tissue and systemic factors. One should note a major limitation of bioimpedance measurements in general is an assumed constant hydration factor (58). Although this is partially addressed through the use of saline swabs prior to impedance measurements, formal evaluation of reliability and accuracy in the setting of inflammatory dermatoses (as opposed to melanocytic lesions) is required. Systematic analytical validation of EIS results in the setting of inflammatory dermatoses would be required prior to definitive statements regarding potential utility in HS.

\section{Optical Coherence Tomography (OCT)}

Optical coherence tomography (OCT) is an interferometric imaging procedure that utilizes the scattering of reflected light to generate a three-dimensional image. It labels the epidermis (particularly in glabrous skin) with high contrast as well as differentiates between papillary and reticular dermis. OCT penetrates to a depth of $1-1.5 \mathrm{~mm}$ and can be viewed enface or as reconstructed three-dimensional images $(12,59)$. OCT accurately distinguishes actinic keratosis from basal cell carcinoma (60) and quantifies psoriasiform epidermal thickening and dermal vascular dilatation in psoriasis (61). OCT has also quantified changes in blood flow in response to topical treatment with brimonidine gel in rosacea (62). The cost of equipment is a potential barrier to widespread adoption of OCT; however, it has the potential for increased accuracy and resolution of epidermal and dermal structures compared with ultrasound. Other potential limitations include the effect of subcutaneous abscesses and scarring in HS upon the accuracy of its readings. Vitreal hemorrhage and exudate can obscure retinal OCT imaging (63) and the presence of fibrotic tissue resulted in false positive readings suggestive of basal cell carcinoma in healthy tissue (64).

\section{Medical Infrared Thermography (MIT)}

Medical infrared thermography (MIT) detects minimal temperature differences between different skin areas based on infrared emissions. It produces a two-dimensional thermal map in which brightness correlates with tissue temperature down to a depth of 2-3 mm (65). MIT has assessed burn wound depth (66) and is being actively investigated as an

TABLE 1 | Potential imaging-based biomarkers identified for further investigation from this scoping review.

\begin{tabular}{|c|c|c|c|}
\hline $\begin{array}{l}\text { Imaging } \\
\text { modality }\end{array}$ & Potential imaging biomarker & Potential disease activity correlate(s) & Potential clinical correlate(s) \\
\hline \multirow[t]{4}{*}{ Ultrasound } & Epidermal Thickness & $\begin{array}{l}\text { IL-23, IL-22, IL-17A, IL-17C, TNF-alpha, } \\
\text { Histologic sections }\end{array}$ & HiSCR, IHS4, AISI, \\
\hline & Dermal/Hypodermal Vascularity & $\begin{array}{l}\text { VEGF, TNF-alpha, } \\
\text { Histologic sections }\end{array}$ & HiSCR, IHS4, AISI, pain score \\
\hline & Dermal/hypodermal Tunnel Diameter & Histologic sections & $\begin{array}{l}\text { Volume of tunnel drainage, IHS4, } \\
\text { Pain Score }\end{array}$ \\
\hline & $\begin{array}{l}\text { Vascularity adjacent to subcutaneous } \\
\text { collections }\end{array}$ & $\begin{array}{l}\text { VEGF, TNF-alpha, } \\
\text { Histologic sections }\end{array}$ & HiSCR, IHS4, AISI, pain score \\
\hline MRI & Dermal/Hypodermal Tunnel Diameter & Histologic sections & $\begin{array}{l}\text { Volume of tunnel drainage, IHS4, } \\
\text { Pain score }\end{array}$ \\
\hline \multirow[t]{3}{*}{$\mathrm{RCM}$} & Dendritic Cell Density & Histologic sections, CD1a, CD207, & HiSCR, IHS4, AISI, \\
\hline & Epidermal Thickness & IL-23, IL-22, IL-17A, IL-17C, histologic sections & HiSCR, IHS4, AISI, \\
\hline & Dermal Papillae Vascularity & $\begin{array}{l}\text { VEGF, TNF-alpha, } \\
\text { Histologic sections }\end{array}$ & HiSCR, IHS4, AISI, Pain score \\
\hline \multirow[t]{3}{*}{ OCT } & Epidermal Thickness & $\begin{array}{l}\text { IL-23, IL-22, IL-17A, IL-17C, Histologic } \\
\text { sections }\end{array}$ & HiSCR, IHS4, AISI, \\
\hline & Dermal Fibrosis & Histologic sections & HiSCR, IHS4, AISI, \\
\hline & Dermal Vascularity & $\begin{array}{l}\text { VEGF, TNF-alpha, } \\
\text { histologic sections }\end{array}$ & HiSCR, IHS4, AISI, Pain score \\
\hline
\end{tabular}


alternative to ultrasound in the grading of HS. MIT has been correlated with qualitative changes in Doppler blood flow in HS (67) and have defined tissue margins in HS skin removal surgery $(67,68)$. It remains unclear whether the accuracy and reproducibility of MIT is influenced by body site, previous surgical excisions and microvascular disease from smoking and/or metabolic syndrome, which may all influence tissue blood flow (69). MIT has shown to have significant variability between sessions and subjects (70), bringing into question the utility of MIT as a longitudinal assessment modality.

\section{Identifying Analytical and Clinical Benchmarks for Future Biomarker Validation}

The results of this scoping review have identified a number of imaging outcomes that have potential to be assessed for analytical and clinical validity in the setting of inflammatory skin diseases such as HS (Table 1). Currently little is known regarding the correlation of imaging findings in HS with known biological markers of disease activity and severity. In lesional and non-lesional tissue, quantification of TNF- $\alpha$, IL-17, and IL-1 $\beta$ mRNA has correlated with HS disease severity $(71,72)$ and response to treatment (34). This raises the possibility that lesional skin mRNA levels can be used as an analytical standard from which to assess the validity of imaging biomarkers. Given the well-established role of inflammation (particularly IL-17) in epidermal changes, including psoriasiform hyperplasia (73), it is reasonable to hypothesize that non-invasive imaging findings would parallel biological and clinical response, albeit in a delayed fashion compared with mRNA and immunohistochemistry. In order to extrapolate imaging biomarkers into surrogate outcomes of clinical response, longitudinal studies and reliable clinical outcome benchmarks are required (2). Given the known

\section{REFERENCES}

1. Hindelang B, Aguirre J, Schwarz M, Berezhnoi A, Eyerich K, Ntziachristos $\mathrm{V}$, et al. Non-invasive imaging in dermatology and the unique potential of raster-scan optoacoustic mesoscopy. J Eur Acad Dermatol Venereol. (2019) 33:1051-61. doi: 10.1111/jdv.15342

2. BEST. BEST (Biomarkers, EndpointS, and other Tools) Resource. Bethesda, MD: Silver Spring (2016).

3. O'Connor JP, Aboagye EO, Adams JE, Aerts HJ, Barrington SF, Beer AJ, et al. Imaging biomarker roadmap for cancer studies. Nat Rev Clin Oncol. (2017) 14:169-86. doi: 10.1038/nrclinonc.2016.162

4. Kimball AB, Ganguli A, Fleischer A. Reliability of the hidradenitis suppurativa clinical response in the assessment of patients with hidradenitis suppurativa. $J$ Eur Acad Dermatol Venereol. (2018) 32:2254-6. doi: 10.1111/jdv.15163

5. Ali AA, Seng EK, Alavi A, Lowes MA. Exploring changes in placebo treatment arms in hidradenitis suppurativa randomized clinical trials: a systematic review. J Am Acad Dermatol. (2019) S0190-9622(19)308692. doi: 10.1016/j.jaad.2019.05.065. [Epub ahead of print].

6. Landeck L, Kneip C, Reischl J, Asadullah K. Biomarkers and personalized medicine: current status and further perspectives with special focus on dermatology. Exp Dermatol. (2016) 25:333-9. doi: 10.1111/exd.12948

7. Pham MT, Rajić A, Greig JD, Sargeant JM, Papadopoulos A, McEwen SA. A scoping review of scoping reviews: advancing the approach and enhancing the consistency. Res Synth Methods. (2014) 5:371-85. doi: 10.1002/jrsm.1123

8. Munn Z, Peters MDJ, Stern C, Tufanaru C, McArthur A, Aromataris E. Systematic review or scoping review? Guidance for authors when choosing variability in HS clinical rating scales, multiple clinical outcome scales may need to be utilized. The need for accurate longitudinal measurements implies that MIT may not be an appropriate imaging modality to develop as a surrogate outcome of clinical response (4).

\section{FUTURE DIRECTIONS}

Currently, ultrasound is the most widely used imaging modality in clinical practice for HS. The low cost and accessibility are aspects that make ultrasound-based imaging biomarkers attractive for future validation. Emerging methods such as OCT and RCM demonstrate higher degrees of accuracy regarding epidermal thickness which may outweigh the great cost. The utility of MRI is largely restricted to pre-operative disease assessment; however, it is the only modality with a proof of concept to be able to measure deep dermal tunnel dilatation as a quantifiable surrogate measure of tunnel drainage. EIS has a number of potential confounding effects that require exploration in the setting of inflammatory skin disease, and MIT is limited by its lack of reproducibility. In summary, we have identified a number of potential imaging-based variables which require further investigation to evaluate their potential as imaging-based biomarkers for HS and discussed their benefits and limitations pertaining to their potential to meet criteria for biomarker validity and reliability.

\section{AUTHOR CONTRIBUTIONS}

DG, KN, and JF were responsible for the concept of the study. DG wrote the first draft of the manuscript. JF and $\mathrm{KN}$ wrote portions of the manuscript. All authors read and approved of the final version of the manuscript. between a systematic or scoping review approach. BMC Med Res Methodol. (2018) 18:143. doi: 10.1186/s12874-018-0611-x

9. Peters MD, Godfrey CM, Khalil H, McInerney P, Parker D, Soares CB. Guidance for conducting systematic scoping reviews. Int J Evid Based Healthc. (2015) 13:141-6. doi: 10.1097/XEB.0000000000000050

10. Wortsman X. Imaging of hidradenitis suppurativa. Dermatol Clin. (2016) 34:59-68. doi: 10.1016/j.det.2015.08.003

11. Halani S, Foster FS, Breslavets $M$, Shear NH. Ultrasound and infrared-based imaging modalities for diagnosis and management of cutaneous diseases. Front Med. (2018) 5:115. doi: 10.3389/fmed.2018. 00115

12. Berardesca E, Maibach HI, K.-Wilhelm P. Non Invasive Diagnostic Techniques in Clinical Dermatology. Berlin; Heidelberg: Springer (2014).

13. Dasgeb B, Kainerstorfer J, Mehregan D, Van Vreede A, Gandjbakhche A. An introduction to primary skin imaging. Int J Dermatol. (2013) 52:1319-30. doi: $10.1111 /$ ijd. 12048

14. El Gammal S, El Gammal C, Altmeyer P, Vogt M. Sonography of the skin. In: Berardesca E, Maibach HI, Wilhelm KP, editors. Non Invasive Diagnostic Techniques in Clinical Dermatology. Berlin; Heidelberg: Springer (2014). p. 135-56.

15. Gupta AK, Turnbull DH, Harasiewicz KA, Shum DT, Watteel GN, Foster FS, et al. The use of high-frequency ultrasound as a method of assessing the severity of a plaque of psoriasis. Arch Dermatol. (1996) 132:65862. doi: 10.1001/archderm.132.6.658

16. Yazdanparast T, Yazdani K, Humbert P, Khatami A, Ahmad Nasrollahi S, Zartab H, et al. Biophysical and ultrasonographic changes in lichen planus 
compared with uninvolved skin. Int J Womens Dermatol. (2019) 5:1004. doi: 10.1016/j.ijwd.2018.10.001

17. el Gammal S, Pieck C, Auer T, Kaspar K, Hoffmann K, Altmeyer P, et al. [100 MHz ultrasound of psoriasis vulgaris plaque]. Ultraschall Med. (1998) 19:270-4. doi: 10.1055/s-2007-1000503

18. Bard RL. High-frequency ultrasound examination in the diagnosis of skin cancer. Dermatol Clin. (2017) 35:505-11. doi: 10.1016/j.det.2017.06.011

19. Lassau N, Koscielny S, Avril MF, Margulis A, Duvillard P, De Baere T, et al. Prognostic value of angiogenesis evaluated with high-frequency and color Doppler sonography for preoperative assessment of melanomas. AJR Am J Roentgenol. (2002) 178:1547-51. doi: 10.2214/ajr.178.6.1781547

20. Lis-Swiety A, Janicka I, Skrzypek-Salamon A, Brzezinska-Wcisło L. A systematic review of tools for determining activity of localized scleroderma in paediatric and adult patients. J Eur Acad Dermatol Venereol. (2017) 31:307. doi: $10.1111 /$ jdv. 13790

21. Osmola-Mankowska A, Silny W, Danczak-Pazdrowska A, Polanska A, Olek-Hrab K, Sadowska-Przytocka A, et al. Assessment of chronic sclerodermoid Graft-versus-Host Disease patients, using $20 \mathrm{MHz}$ highfrequency ultrasonography and cutometer methods. Skin Res Technol. (2013) 19:e417-22. doi: 10.1111/j.1600-0846.2012.00659.x

22. Chew PG, Bounford K, Plein S, Schlosshan D, Greenwood JP. Multimodality imaging for the quantitative assessment of mitral regurgitation. Quant Imaging Med Surg. (2018) 8:342-59. doi: 10.21037/qims.2018.04.01

23. Singh G, Kumar P, Parshad R, Seith A, Thulkar S, Hosten N. Role of color Doppler indices in predicting disease-free survival of breast cancer patients during neoadjuvant chemotherapy. Eur J Radiol. (2010) 75:e15862. doi: 10.1016/j.ejrad.2009.12.027

24. Kruskal JB, Newman PA, Sammons LG, Kane RA. Optimizing Doppler and color flow US: application to hepatic sonography. Radiographics. (2004) 24:657-75. doi: 10.1148/rg.243035139

25. Kelekis NL, Efstathopoulos E, Balanika A, Spyridopoulos TN, Pelekanou A, Kanni T, et al. Ultrasound aids in diagnosis and severity assessment of hidradenitis suppurativa. Br J Dermatol. (2010) 162:1400-2. doi: 10.1111/j.1365-2133.2010.09710.x

26. van der Zee HH, de Ruiter L, Boer J, van den Broecke DG, den Hollander JC, Laman JD, et al. Alterations in leucocyte subsets and histomorphology in normal-appearing perilesional skin and early and chronic hidradenitis suppurativa lesions. Br J Dermatol. (2012) 166:98106. doi: 10.1111/j.1365-2133.2011.10643.x

27. Wortsman X, Moreno C, Soto R, Arellano J, Pezo C, Wortsman J. Ultrasound in-depth characterization and staging of hidradenitis suppurativa. Dermatol Surg. (2013) 39:1835-42. doi: 10.1111/dsu.12329

28. Nazzaro G, Passoni E, Calzari P, Barbareschi M, Muratori S, Veraldi S, et al. Color Doppler as a tool for correlating vascularization and pain in hidradenitis suppurativa lesions. Skin Res Technol. (2019). doi: 10.1111/srt.12729. [Epub ahead of print].

29. Zarchi K, Yazdanyar N, Yazdanyar S, Wortsman X, Jemec GB. Pain and inflammation in hidradenitis suppurativa correspond to morphological changes identified by high-frequency ultrasound. J Eur Acad Dermatol Venereol. (2015) 29:527-32. doi: 10.1111/jdv.12610

30. Martorell A, Alfageme Roldán F, Vilarrasa Rull E, Ruiz-Villaverde R, Romaní De Gabriel J, García Martínez F, et al. Ultrasound as a diagnostic and management tool in hidradenitis suppurativa patients: a multicenter study. J Eur Acad Dermatol Venereol. (2019). doi: 10.1111/jdv.15710. [Epub ahead of print].

31. Martorell A, Giovanardi G, Gomez-Palencia P, Sanz-Motilva V. Defining fistular patterns in hidradenitis suppurativa: impact in the management. Dermatol Surg. (2019) 45:1237-44. doi: 10.1097/DSS.0000000 000001916

32. Lyons AB, Zubair R, Kohli I, Hamzavi IH. Preoperative ultrasound for evaluation of hidradenitis suppurativa. Dermatol Surg. (2019) 45:2946. doi: 10.1097/DSS.0000000000001696

33. Kelly G, Hughes R, McGarry $\mathrm{T}$, van den Born M, Adamzik K, Fitzgerald R, et al. Dysregulated cytokine expression in lesional and nonlesional skin in hidradenitis suppurativa. Br J Dermatol. (2015) 173:14319. doi: $10.1111 /$ bjd.14075

34. Moran B, Sweeney CM, Hughes R, Malara A, Kirthi S, Tobin AM, et al. Hidradenitis suppurativa is characterized by dysregulation of the Th17:treg cell axis, which is corrected by anti-TNF therapy. J Invest Dermatol. (2017) 137:2389-95. doi: 10.1016/j.jid.2017.05.033

35. Schanz S, Fierlbeck G, Ulmer A, Schmalzing M, Kümmerle-Deschner J, Claussen CD, et al. Localized scleroderma: MR findings and clinical features. Radiology. (2011) 260:817-24. doi: 10.1148/radiol.11102136

36. Lim R, Jaramillo D, Poussaint TY, Chang Y, Korf B. Superficial neurofibroma: a lesion with unique MRI characteristics in patients with neurofibromatosis type 1. AJR Am J Roentgenol. (2005) 184:962-8. doi: 10.2214/ajr.184.3.01840962

37. Mamlouk MD, Nicholson AD, Cooke DL, Hess CP. Tips and tricks to optimize MRI protocols for cutaneous vascular anomalies. Clin Imaging. (2017) 45:7180. doi: 10.1016/j.clinimag.2017.05.019

38. Querleux B. Multimodal magnetic resonance imaging of the human skin, in non invasive diagnostic techniques in clinical dermatology. In: Berardesca E, Maibach HI, Wilhelm KP, editors. Non Invasive Diagnostic Techniques in Clinical Dermatology. Berlin; Heidelberg: Springer (2014). p. 169-176. doi: 10.1007/978-3-642-32109-2_15

39. Balci S, Onur MR, Karaosmanoglu AD, Karçaaltincaba M, Akata D, Konan A, et al. MRI evaluation of anal and perianal diseases. Diagn Interv Radiol. (2019) 25:21-7. doi: 10.5152/dir.2018.17499

40. Kelly AM, Cronin P. MRI features of hidradenitis suppurativa and review of the literature. AJR Am J Roentgenol. (2005) 185:1201-4. doi: 10.2214/AJR.04.1233

41. Griffin N, Williams AB, Anderson S, Irving PM, Sanderson J, Desai N, et al. Hidradenitis suppurativa: MRI features in anogenital disease. Dis Colon Rectum. (2014) 57:762-71. doi: 10.1097/DCR.0000000000000131

42. Kimball AB, Okun MM, Williams DA, Gottlieb AB, Papp KA, Zouboulis CC, et al. Two phase 3 trials of adalimumab for hidradenitis suppurativa. $N$ Engl $J$ Med. (2016) 375:422-34. doi: 10.1056/NEJMoa 1504370

43. Zouboulis CC, Tzellos T, Kyrgidis A, Jemec GBE, Bechara FG, GiamarellosBourboulis EJ, et al. Development and validation of the International Hidradenitis Suppurativa Severity Score System (IHS4), a novel dynamic scoring system to assess HS severity. Br J Dermatol. (2017) 177:14019. doi: $10.1111 /$ bjd. 15748

44. Chiricozzi A, Faleri S, Franceschini C, Caro RD, Chimenti S, Bianchi L. AISI: a new disease severity assessment tool for hidradenitis suppurativa. Wounds. (2015) 27:258-64.

45. Schneider SL, Kohli I, Hamzavi IH, Council ML, Rossi AM, Ozog DM. Emerging imaging technologies in dermatology: part I: basic principles. J Am Acad Dermatol. (2019) 80:1114-20. doi: 10.1016/j.jaad.2018.11.042

46. Webber SA, Wurm EM, Douglas NC, Lambie D, Longo C, Pellacani G, et al. Effectiveness and limitations of reflectance confocal microscopy in detecting persistence of basal cell carcinomas: a preliminary study. Australas J Dermatol. (2011) 52:179-85. doi: 10.1111/j.1440-0960.2011.00769.x

47. Batani A, Brănişteanu DE, Ilie MA, Boda D, Ianosi S, Ianosi G, et al. Assessment of dermal papillary and microvascular parameters in psoriasis vulgaris using in vivo reflectance confocal microscopy. Exp Ther Med. (2018) 15:1241-6. doi: 10.3892/etm.2017.5542

48. Ardigò M, Agozzino M, Longo C, Lallas A, Di Lernia V, Fabiano A, et al. Reflectance confocal microscopy for plaque psoriasis therapeutic follow-up during an anti-TNF-alpha monoclonal antibody: an observational multicenter study. J Eur Acad Dermatol Venereol. (2015) 29:23638. doi: 10.1111/jdv.13235

49. Manfredini M, Mazzaglia G, Ciardo S, Farnetani F, Mandel VD, Longo C, et al. Acne: in vivo morphologic study of lesions and surrounding skin by means of reflectance confocal microscopy. J Eur Acad Dermatol Venereol. (2015) 29:933-9. doi: $10.1111 / j \mathrm{jv} .12730$

50. Manfredini M, Greco M, Farnetani F, Mazzaglia G, Ciardo S, Bettoli V, et al. In vivo monitoring of topical therapy for acne with reflectance confocal microscopy. Skin Res Technol. (2017) 23:36-40. doi: 10.1111/srt. 12298

51. Mancebo SE, Cordova M, Myskowski PL, Flores ES, Busam K, Jawed SI, et al. Reflectance confocal microscopy features of mycosis fungoides and Sezary syndrome: correlation with histopathologic and T-cell receptor rearrangement studies. J Cutan Pathol. (2016) 43:505-15. doi: 10.1111/cup.12708

52. von Laffert M, Helmbold P, Wohlrab J, Fiedler E, Stadie V, Marsch WC. Hidradenitis suppurativa (acne inversa): early inflammatory events 
at terminal follicles and at interfollicular epidermis. Exp Dermatol. (2010) 19:533-7. doi: 10.1111/j.1600-0625.2009.00915.x

53. Braun RP, Mangana J, Goldinger S, French L, Dummer R, Marghoob AA. Electrical impedance spectroscopy in skin cancer diagnosis. Dermatol Clin. (2017) 35:489-93. doi: 10.1016/j.det.2017.06.009

54. Svoboda RM, Prado G, Mirsky RS, Rigel DS. Assessment of clinician accuracy for diagnosing melanoma on the basis of electrical impedance spectroscopy score plus morphology versus lesion morphology alone. J Am Acad Dermatol. (2019) 80:285-7. doi: 10.1016/j.jaad.2018.08.048

55. Milani GP, Groothoff JW, Vianello FA, Fossali EF, Paglialonga F, Edefonti A, et al. Bioimpedance and fluid status in children and adolescents treated with dialysis. Am J Kidney Dis. (2017) 69:428-35. doi: 10.1053/j.ajkd.2016.10.023

56. Gerosa M, Zimlichman E, Ventura D, Fanelli V, Riboldi P, Meroni PL. Measurement of electrical skin impedance of dermal-visceral zones as a diagnostic tool for disorders of the immune system. Lupus. (2006) 15:45761. doi: 10.1191/0961203306lu2333oa

57. Dittmar M, Reber H, Kahaly GJ. Bioimpedance phase angle indicates catabolism in Type 2 diabetes. Diabet Med. (2015) 32:1177-85. doi: $10.1111 /$ dme.12710

58. Deurenberg P. Limitations of the bioelectrical impedance method for the assessment of body fat in severe obesity. Am J Clin Nutr. (1996) 64(3 Suppl.):449S-52S. doi: 10.1093/ajcn/64.3.449S

59. Welzel J, Schuh S. Noninvasive diagnosis in dermatology. J Dtsch Dermatol Ges. (2017) 15:999-1016. doi: 10.1111/ddg.13347

60. Schuh S, Kaestle R, Sattler EC, Welzel J. Optical coherence tomography of actinic keratoses and basal cell carcinomas - differentiation by quantification of signal intensity and layer thickness. J Eur Acad Dermatol Venereol. (2016) 30:1321-6. doi: 10.1111/jdv.13569

61. Morsy H, Kamp S, Thrane L, Behrendt N, Saunder B, Zayan H, et al. Optical coherence tomography imaging of psoriasis vulgaris: correlation with histology and disease severity. Arch Dermatol Res. (2010) 302:10511. doi: 10.1007/s00403-009-1000-4

62. Themstrup L, Ciardo S, Manfredi M, Ulrich M, Pellacani G, Welzel $\mathrm{J}$, et al. In vivo, micro-morphological vascular changes induced by topical brimonidine studied by Dynamic optical coherence tomography. J Eur Acad Dermatol Venereol. (2016) 30:974-9. doi: 10.1111/jdv. 13596

63. Huang YM, Hsieh MH, Li AF, Chen SJ. Sensitivity, specificity, and limitations of optical coherence tomography angiography in diagnosis of polypoidal choroidal vasculopathy. J Ophthalmol. (2017) 2017:3479695. doi: 10.1155/2017/3479695

64. Alawi SA, Kuck M, Wahrlich C, Batz S, McKenzie G, Fluhr JW, et al. Optical coherence tomography for presurgical margin assessment of nonmelanoma skin cancer - a practical approach. Exp Dermatol. (2013) 22:54751. doi: $10.1111 /$ exd.12196
65. Gurjarpadhye AA, Parekh MB, Dubnika A, Rajadas J, Inayathullah M. Infrared imaging tools for diagnostic applications in dermatology. SM J Clin Med Imaging. (2015) 1:1-5.

66. Medina-Preciado JD, Kolosovas-Machuca ES, Velez-Gomez E, MirandaAltamirano A, González FJ. Noninvasive determination of burn depth in children by digital infrared thermal imaging. J Biomed Opt. (2013) 18:061204. doi: 10.1117/1.JBO.18.6.061204

67. Nazzaro G, Moltrasio C, Marzano AV. Infrared thermography and color Doppler: two combined tools for assessing inflammation in hidradenitis suppurativa. Skin Res Technol. (2019). doi: 10.1111/srt.12750. [Epub ahead of print].

68. Zouboulis CC, Nogueira da Costa A, Jemec GBE, Trebing D. Longwave medical infrared thermography: a clinical biomarker of inflammation in hidradenitis suppurativa/acne inversa. Dermatology. (2019) 235:1449. doi: $10.1159 / 000495982$

69. Chojnowski M. Infrared thermal imaging in connective tissue diseases. Reumatologia. (2017) 55:38-43. doi: 10.5114/reum.2017.66686

70. Christensen J, Vaeth M, Wenzel A. Thermographic imaging of facial skingender differences and temperature changes over time in healthy subjects. Dentomaxillofac Radiol. (2012) 41:662-7. doi: 10.1259/dmfr/55922484

71. Witte-Händel E, Wolk K, Tsaousi A, Irmer ML, Mößner R, Shomroni O, et al. The IL-1 pathway is hyperactive in hidradenitis suppurativa and contributes to skin infiltration and destruction. J Invest Dermatol. (2019) 139:1294305. doi: 10.1016/j.jid.2018.11.018

72. van der Zee HH, de Ruiter L, van den Broecke DG, Dik WA, Laman JD, Prens EP. Elevated levels of tumour necrosis factor (TNF)-alpha, interleukin (IL)-1beta and IL-10 in hidradenitis suppurativa skin: a rationale for targeting TNF-alpha and IL-1beta. Br J Dermatol. (2011) 164:12928. doi: 10.1111/j.1365-2133.2011.10254.x

73. Lima AL, Karl I, Giner T, Poppe H, Schmidt M, Presser D, et al. Keratinocytes and neutrophils are important sources of proinflammatory molecules in hidradenitis suppurativa. Br J Dermatol. (2016) 174:51421.doi: $10.1111 /$ bjd. 14214

Conflict of Interest: The authors declare that the research was conducted in the absence of any commercial or financial relationships that could be construed as a potential conflict of interest.

Copyright (๑) 2019 Grand, Navrazhina and Frew. This is an open-access article distributed under the terms of the Creative Commons Attribution License (CC BY). The use, distribution or reproduction in other forums is permitted, provided the original author(s) and the copyright owner(s) are credited and that the original publication in this journal is cited, in accordance with accepted academic practice. No use, distribution or reproduction is permitted which does not comply with these terms. 